\title{
Collaborative engineering and design management for the Hobby- Eberly Telescope tracker upgrade
}

\author{
Nicholas T. Mollison ${ }^{\dagger \mathrm{a}}$, Richard J. Hayes ${ }^{\mathrm{a}}$, John M. Good ${ }^{\mathrm{b}}$, John A. Booth ${ }^{\mathrm{b}}$, Richard D. Savage ${ }^{\mathrm{b}}$, \\ John R. Jackson ${ }^{\mathrm{a}}$, Marc D. Rafal ${ }^{\mathrm{b}}$, Joseph H. Beno ${ }^{\mathrm{a}}$ \\ ${ }^{a}$ The University of Texas at Austin, Center for Electromechanics, 1 University Station R7000, \\ Austin, Texas USA 78712 \\ ${ }^{\mathrm{b}}$ The University of Texas McDonald Observatory, 1 University Station C1402, Austin, Texas, USA \\ 78712-0259;
}

\begin{abstract}
The engineering and design of systems as complex as the Hobby-Eberly Telescope's* new tracker require that multiple tasks be executed in parallel and overlapping efforts. When the design of individual subsystems is distributed among multiple organizations, teams, and individuals, challenges can arise with respect to managing design productivity and coordinating successful collaborative exchanges. This paper focuses on design management issues and current practices for the tracker design portion of the Hobby-Eberly Telescope Wide Field Upgrade project. The scope of the tracker upgrade requires engineering contributions and input from numerous fields including optics, instrumentation, electromechanics, software controls engineering, and site-operations. Successful system-level integration of tracker subsystems and interfaces is critical to the telescope's ultimate performance in astronomical observation. Software and process controls for design information and workflow management have been implemented to assist the collaborative transfer of tracker design data. The tracker system architecture and selection of subsystem interfaces has also proven to be a determining factor in design task formulation and team communication needs. Interface controls and requirements change controls will be discussed, and critical team interactions are recounted (a group-participation Failure Modes and Effects Analysis [FMEA] is one of special interest). This paper will be of interest to engineers, designers, and managers engaging in multi-disciplinary and parallel engineering projects that require coordination among multiple individuals, teams, and organizations.
\end{abstract}

Keywords: Center for Electromechanics, CEM, Hobby-Eberly Telescope, HETDEX, University of Texas, parallel engineering, engineering management, project management

\section{INTRODUCTION}

\subsection{Background}

The Hobby-Eberly Telescope (HET) is undergoing a major upgrade in preparation for the HET Dark Energy eXperiment* (HETDEX), as well as to enhance support capabilities for current and future instrumentation ${ }^{1}$. HETDEX entails two major hardware upgrades to the HET: the Wide Field Upgrade (WFU), and the fabrication and installation of up to 192 spectrometers called VIRUS ${ }^{2}$. The WFU consists of four related engineering projects: 1) Replacing the current spherical aberration corrector with the Wide Field Corrector ${ }^{3}$ (WFC), which will increase the HET field of view from 4' to $\left.22^{\prime}, 2\right)$ replacing the entire tracker system, 3) a new Prime Focus Instrument Package (PFIP), and 4) modifications to the HET facility ${ }^{1}$. Under the tracker portion of the program, improvements are being made to the HET's motion control systems to increase tracker payload capacity and to hasten "reset" speed, allowing more observations per night as required for HETDEX ${ }^{2}$.

* The Hobby-Eberly Telescope is operated by McDonald Observatory on behalf of the University of Texas at Austin, the Pennsylvania State University, Stanford University, Ludwig-Maximillians-Universität München, and Georg-AugustUniversität, Göttingen

$\dagger$ R.S.: email: n.mollison@cem.utexas.edu

$\$$ http://hetdex.org/ 
Developing and engineering systems as complex as those required for HETDEX and the WFU (Figure 1) within a span of less than four years from start through production, testing, installation, and commissioning, is only one factor driving the need for parallel design efforts. Tightly woven subsystem functions and interface requirements have made parallel and collaborative design a necessity due to the "chicken and egg" issues encountered in large-scale system design. There are many instances where one subsystem cannot be fully designed without prerequisite knowledge of another, nor can one design be substantially altered without reflexively impacting another.

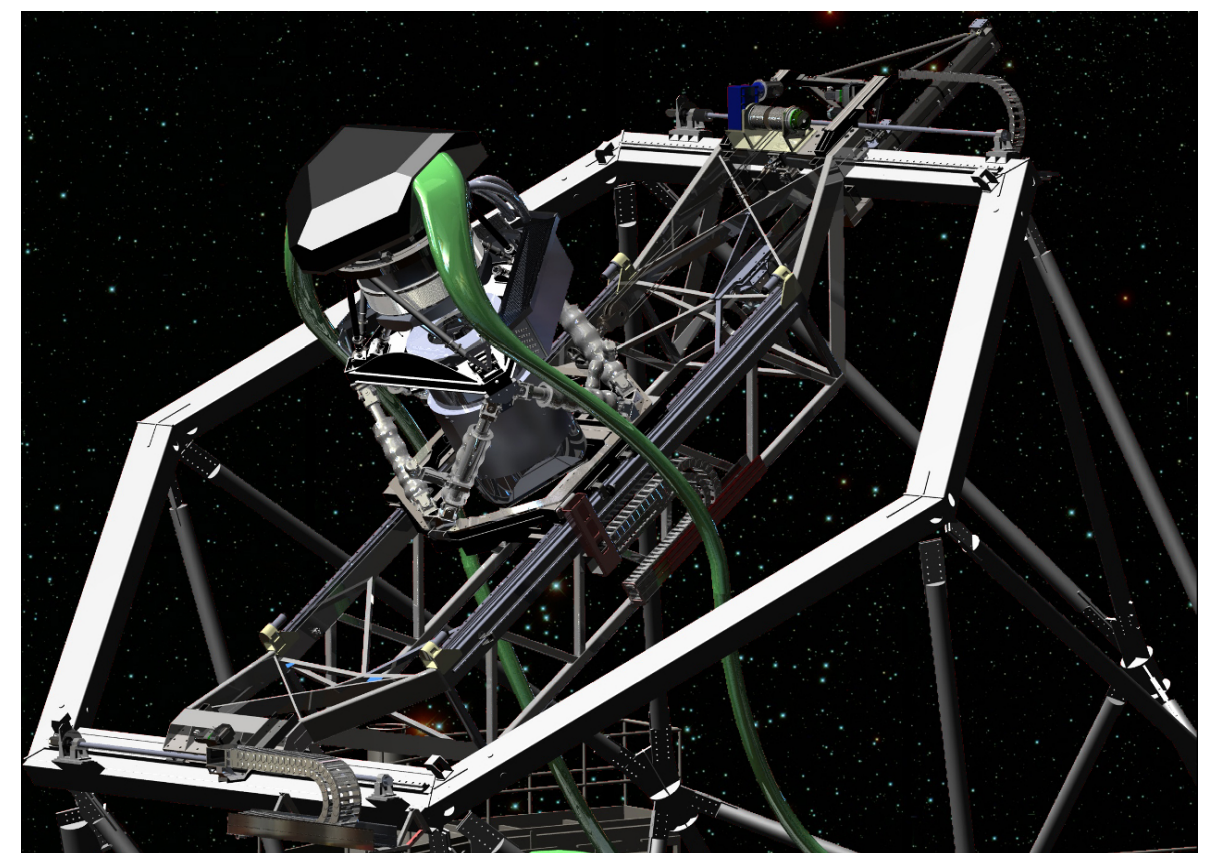

Figure 1. The Hobby-Eberly Telescope tracker design for the Wide Field Upgrade merges numerous contributions from astronomers, engineers, faculty, and many other sources to advance the HET's technological state of the art.

Collaborative engineering is becoming increasingly more prevalent and important to projects that include significant research and development. For any technical manager involved with the design of complex systems or processes, directing a collaborative design effort poses difficult challenges. It is vital for design managers to have a sound grasp on several issues specific to the collaborative environment, as societal trends will continue to increase the scope and necessity of inter and intra-organizational alliances in order to cultivate technological advancement from diverse technical disciplines. The goal of design managers is to maintain a high level of design productivity, enable high-quality design solutions, and orchestrate efficient development cycles. The obstacle to achieving this goal, posed by the parallel collaborative environment, is that design decisions must be made with less than complete information as co-dependent systems and subsystems evolve to reveal new information concurrently.

The scope of the Wide Field Upgrade requires engineering expertise from several disciplines, and from groups that are dispersed among separate organizations and, in some cases, spread geographically around the globe. There were several design requirements and constraints either still evolving or yet to be determined at the onset of the program, some of which could not be established without first developing preliminary concept designs. Time-to-market pressure has also been present in the form of completing the Dark Energy eXperiment quickly to deliver its extraordinary scientific impact, and to promulgate the project's engineering achievements to benefit similar programs within the scientific community. Finally, significant design consideration has been granted to reliability, maintainability, and manufacturability of the various tracker components. To satisfy all design objectives within the environment described above, it can be inferred that a substantial degree of collaboration is required among design participants. Accounts of noteworthy interactions pertaining to the parallel efforts of the parties involved will be discussed in subsequent sections of this paper. The aim of this paper is to illuminate a few key factors that have far-reaching influence over the collaborative design project outcome, accompanied by management practices and lessons learned on the tracker upgrade project. These factors include the choices made when dividing subsystem interfaces to form the system architecture, the exchange of design information, and issues that arise from growing system complexity. 


\section{SYSTEM ARCHITECTURE}

System architecture choices may not be immediately associated with communication needs and constraints, but it will be shown that architecture is a deterministic factor for the information transfer needs of a collaborative project. System architecture refers to the structural arrangement of the system being designed and, more specifically, the division of the system into subsystems and their resulting connectivity and interactions. Dividing a system into functional blocks, subsystem modules, and even further into component sets is what permits parallel engineering to occur, such that the work can be divided amongst various groups who will collaborate to shepherd modules through the design process simultaneously. The division of work responsibilities, however, is not necessarily the underlying motivator for engaging in collaborative exchanges. Collaboration is required by the fact that each of the subsystems and components that form the system architecture must share compatibility at their interfaces for the system to fulfill its intended function. Each division of the system creates at least one interface between components. In order for the system to work, the components must share at least one type of compatibility with respect to their intended interaction; often in the form of mechanical couplings, electrical interconnects, communication protocol, or data format, to give some examples. Creation of interfaces generates the need for designers to share detailed design information in order to assure such compatibility. Technical and social complexity are each a product of the number of interfaces in the system architecture and the number of peers in the collaborative network. Increasing complexity will have the effect of making decision outcomes within each realm less transparent and predictable a priori. Therefore, the social framework produced by system partitions warrants equal attention alongside technical matters.

\subsection{Tracker design participants}

The tracker is divided into several electronic and mechanical or opto-mechanical subsystems, some of which are standalone and several of which overlap in terms of function or integration. The design of each subsystem and its individual components is the responsibility of an organization or individual within an organization (Figure 2). The McDonald Observatory (MDO) team, under the astronomy department of the College of Natural Sciences at the University of Texas at Austin, is the organization leading HETDEX and the WFU program. MDO also retains responsibility for the design of several instrumentation components, telescope controls, site modifications, and components within the PFIP. They have contracted the Center for Electromechanics (CEM) at the University of Texas at Austin to undertake the design and manufacture of the new tracker, a support system for HETDEX fiber optic bundles ${ }^{4}$, and a support structure for VIRUS 5 . MDO has also contracted the University of Arizona Optical Sciences Center to design and produce the WFC. Further subdivision among organizations occurs within the tracker at the hexapod, for which CEM has subcontracted ADS International of Lecco, Italy to design and manufacture the hexapod actuators while CEM retains responsibility for the hexapod control system. Other collaborations within the project include, but are not limited to: Texas A\&M University, Astrophysikalisches Institut Potsdam of Germany, and members of the Southern African Large Telescope (SALT) engineering staff.

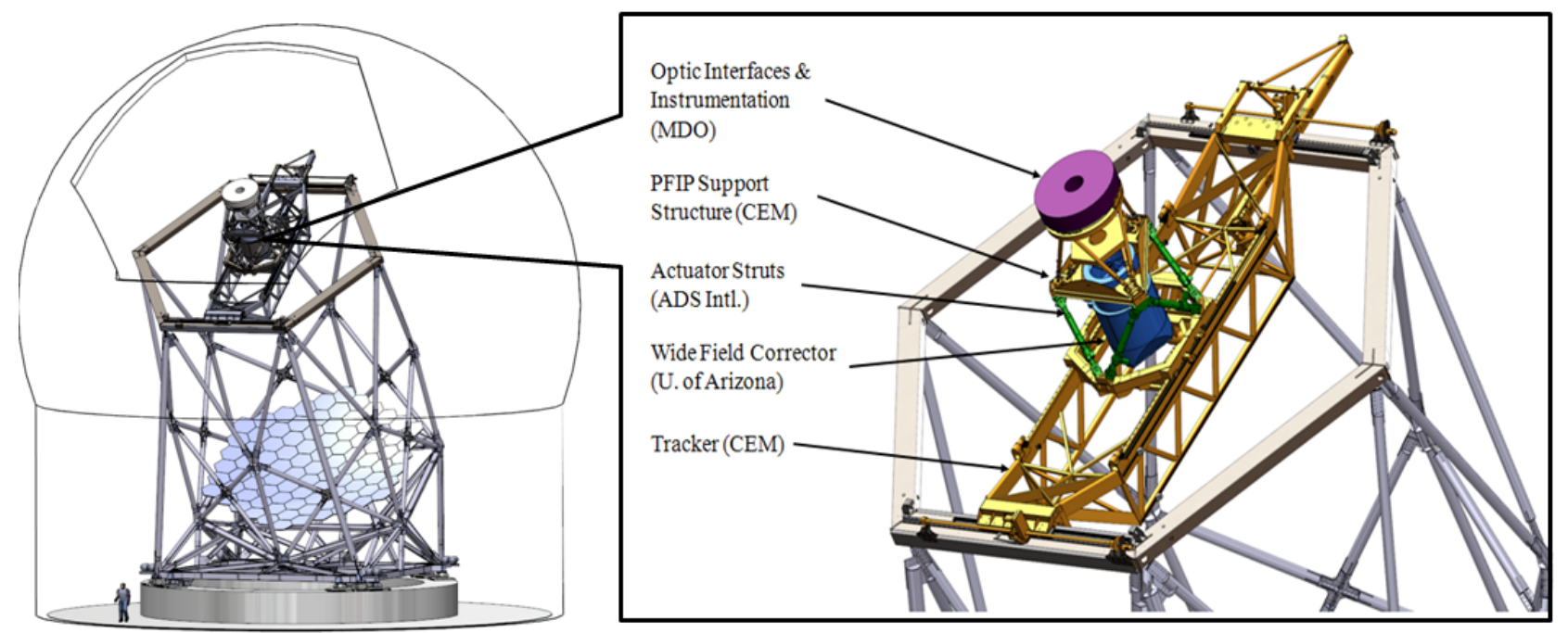

Figure 2. Division of major subsystems and interfaces onboard the tracker by organization for the tracker upgrade. 


\subsection{Tracker system interfaces}

Figure 3 provides a schematic representation of the interfaces contained within the tracker. The partitioning of the upgraded tracker system is a product of several design influences. This new version of the tracker will effectively be the third-generation of the tracker device ${ }^{6}$. The first was designed and constructed for the HET which entered service in the late 1990's. The second was produced for SALT, a cousin of the HET that shares its functional design, but incorporates many improvements enabled by the experience gained operating the HET throughout the time separating the construction of each telescope.

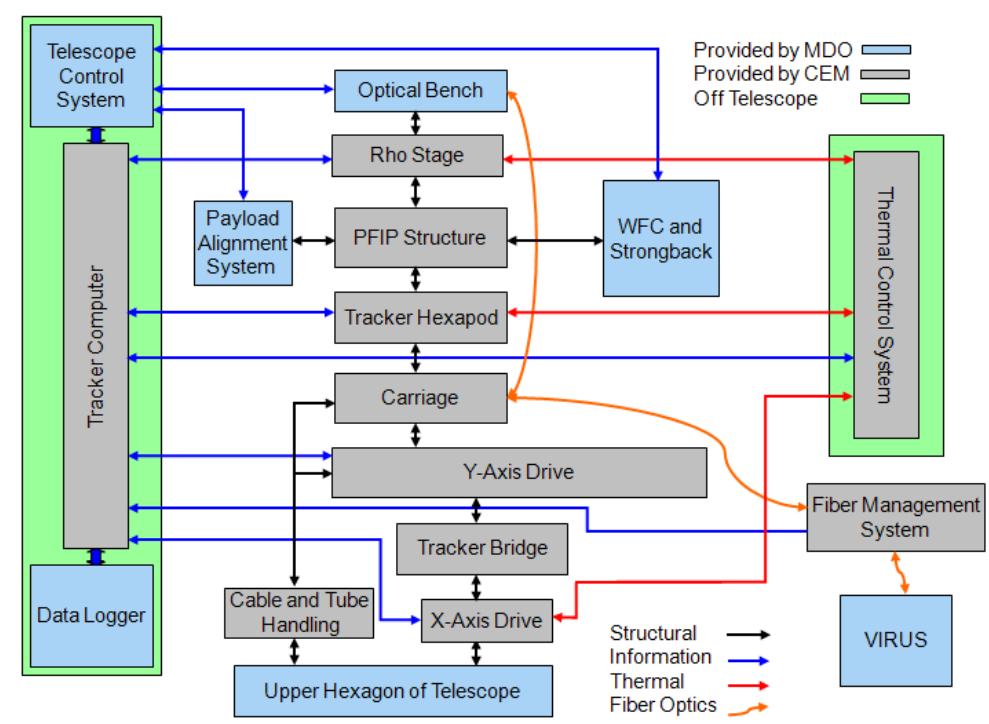

Figure 3. Schematic diagram of the HET's major subsystem interfaces.

Many of the tracker's critical interfaces became well-defined over the course of engineering and designing the two predecessors. For example, the functional interfaces between the tracker's main structural member (the tracker bridge) and its positioning servo-drives are common between all three examples. Other interfaces reflect the differences in specific domain knowledge required to engineer certain subsystems. The interface separating the WFC and the strongback support structure which links it to the tracker is one example. CEM specializes in the electromechanical actuation and controls engineering required for the design of the tracker's motion systems which position the WFC. The WFC is contracted to the University of Arizona Optical Sciences Center for their depth of experience and expertise designing and manufacturing large mirror assemblies for ground and space-borne telescopes. In the instances where major interfaces were known beforehand, and for those interfaces that link subsystems under the charge of organizationally or geographically separated groups (therefore requiring more explicitly detailed and formal communication), an Interface Control Document (ICD) was identified and created in the earliest stages of the project. Table 1 lists the major ICD's, and exemplifies the variety of mechanical, electrical, thermal, pneumatic, and data interfaces that are typical of sophisticated telescope systems.

Table 1. List of current Tracker Interface Control Documents. Each document, which is subject to engineering revision controls, contains a full description of the interface and lists the associated drawings and specifications.

\begin{tabular}{|l|l|}
\hline \multicolumn{1}{|c|}{ Document \# } & \multicolumn{1}{c|}{ Interface Described } \\
\hline HX0039-01-01 & Upper Hexagon of Telescope Structure to Tracker Bridge \\
\hline HX0040-01-01 & Instrumentation Electronics Mounted to Strongback \\
\hline HX0041-01-01 & Hexapod Actuator Mounts to Strongback and Lower Hexapod Frame \\
\hline HX0042-01-01 & Wide Field Corrector Mount to Strongback \\
\hline HX0043-01-01 & Pupil Assembly Platform to Pupil Assembly Instruments \\
\hline HX0044-01-01 & Rho Stage to Focal Plane Assembly Substructure \\
\hline HX0045-01-01 & Tracker Computer to Telescope Computer System \\
\hline HX0046-01-01 & Tracker Electrical Interfaces and Routing Control Document \\
\hline HX0047-01-01 & Tracker Pneumatic Interfaces and Routing Control Document \\
\hline HX0048-01-01 & Tracker Thermal Management System Interfaces and Routing Control Document \\
\hline
\end{tabular}


Structural complexity (referring to the number of system elements and interdependent relationships) generated by the system architecture is relatively easy to manage with respect to the well defined and pre-established interfaces that separate the major subsystem functions of the tracker. However, a clear picture of architectural implications becomes more difficult to sustain as interfaces penetrate deeper into subsystems and finally reach the component level. At lower levels of subsystem design, more latitude exists for interface choices at the behest of individual designers, and likewise so does uncertainty with respect to their details and management of changes.

Figure 2 illustrated a few of the major subsystems and interfaces, as well as the parties responsible for the design of each, but the list of collaborating stakeholders and respective interests that contribute to structural and social complexity does not end there. Research and development projects, and especially those which are marked by a measure of rarity in scope and ambition, often seek to maximize the "one-time opportunity" to enact sweeping enhancements. What is meant by this statement, is that tremendous efforts will be made to incorporate the benefits of direct and indirect experiences; and to incorporate as many technological advancements and latest practices developed in other similar projects as possible so that the design will surpass those that came before it to become the new benchmark. This becomes especially important when the project involves a unique scientific research asset like the Hobby-Eberly Telescope. Therefore, the engineering objectives for the tracker stretch beyond simply upgrading its performance and load capacity. Stakeholders include HET and MDO oversight boards, university consortia, and more directly involved participants such as astronomical research scientists, faculty, operations personnel, and technical staff. Significant communication and design input is required from these groups to optimize the future reliability, maintainability, and ultimately the long-term research value that the HET will provide following the WFU, and for at least another twenty years of operation.

In practical terms, exercising design freedom within subsystems and facilitating the interests of a large number of constituents can create many additional interfaces which may be introduced at virtually any phase of the design process. As a consequence, it is difficult to subject these ancillary interfaces to the same rigorous analysis, formal definition, and revision control scrutiny that high-level system interfaces receive. Yet, the effect they may have both individually and cumulatively on the overall project outcome may still be measurable due to their introduction of new design constraints and dependencies, thereby expanding the system's overall complexity.

\subsection{Managing design dependency}

Task dependency is a familiar concept within Gantt, Program Evaluation and Review Technique (PERT), and Critical Path Method (CPM) project management tools. Design dependency often correlates with task dependency, but subtle differences between the two should be noted in the context of collaborative project planning. Task dependency pertains to discrete logic relationships between the start and completion of specific tasks, and is typically assigned for the purpose of forecasting schedules and resources in a unidirectional progression of tasks over the time duration of the project. Design dependency pertains to the strength of design correlation and direction of design information flow across an interface in the context of the design cycle. For example, component A may pass design information to component $\mathrm{B}$, or vice versa. If information must pass from both $\mathrm{A}$ to $\mathrm{B}$ and $\mathrm{B}$ to $\mathrm{A}$, the design cycle may become iterative. To determine whether or not this is true, a value may be assigned to the strength of design dependency based on the impact changes at the interface may have on each component. If changes to the interface of component $\mathrm{A}$ have almost no effect on component $\mathrm{B}$, the correlation is negligible. If any minor change to the interface of component $\mathrm{A}$ necessitates significant changes to the design of component $\mathrm{B}$, the correlation is a full 1:1 ratio. When information passes to and from both components and the dependency correlation is strong, and a substantial degree of uncertainty also exists within the interface of at least one of them, the design cycle will become highly iterative. This view of design dependency may be used to supplement traditional project planning methods when determining task schedule variability for collaborative tasks. Furthermore, it will be shown in section three of this paper that this method may also be used to coordinate appropriate methods for information exchange.

Design dependencies should be evaluated any time a new interface is proposed. When iterative dependencies are identified, strategies may be employed to manage the extent of rework engendered by subsequent design iterations. Two examples of such strategies can be drawn from the tracker upgrade project. The first is the inclusion of uncertainty margins in design specifications. Margins are set at their maximum at the onset of the project when design variables are least certain, and diminish steadily as designs evolve into finished products. Preliminary design of the tracker bridge was aided by uncertainty margins. The tracker "payload," comprising the PFIP, WFC, hexapod, etc., passes design information to the bridge, but the development of some subsystems within the payload would continue until well-after 
the bridge's release for manufacturing date. Specifically, the payload mass is the most critical piece of interface information relative to the structural design and performance of the bridge. A margin of $20 \%$ was added to the estimated payload mass budget throughout the period leading up to the tracker preliminary design review. By the time of the tracker critical design review, the margin had been reduced to $10 \%$. Although specifying larger uncertainty margins would further reduce the probability of rework later in the design cycle, care must be taken not to set excessive margins that will produce inefficient or poorly optimized designs.

The second strategy for mitigating dependencies is the creation of "buffer" interfaces. Returning to the example of components $\mathrm{A}$ and $\mathrm{B}$ which shared a 1:1 interface correlation, an alternative would be to introduce component $\mathrm{C}$ such that A-C and B-C share 1:1 correlation, but the interfaces of $\mathrm{A}$ and $\mathrm{B}$ are now isolated from each other by component $\mathrm{C}$. Now, no matter how much uncertainty exists at the interface, parallel design of A and B can commence with minimal iteration and rework. Obviously, this solution is not applicable in every scenario, and it has the drawback of creating a greater quantity of components or assemblies, but it is beneficial when the information crossing and interface is limited and design uncertainty can therefore be segregated into inexpensive and easily designed components. Within the tracker design, the Lower Hexapod Frame (LHF) is a complex and multi-functional welded structure under the stewardship of CEM in Austin, Texas. ADS International of Italy is responsible for the hexapod actuator struts which were initially intended to attach directly to the LHF. Due to a later start for the hexapod strut design and geographic communication boundaries, this is a scenario where variability could have been high and means to resolve uncertainty were met with limitations. The Hexapod Plate component was added (for multiple reasons, the others being tangent to this discussion) and additional interfaces were created as a result, but doing so also meant the co-dependencies between the interfaces of the LHF and actuator struts were relieved. The dependency on variable component interfaces was then confined to one simple and easy to change component design and parallel engineering of the LHF and hexapod struts could resume independently.

\subsection{Management considerations regarding system architecture}

Collaborative engagements contribute to the complexity and uncertainty of the design process. As complexity increases, so does the designer's need for information and thus communication. The design team itself becomes a complex system with its own requirements, dependencies, and objectives. Yet, collaboration and uncertainty are necessary to the design process to bring synergistic capabilities to bear and spawn innovation. Designing a new system for the first time often requires preliminary development of subsystem components in parallel to generate the contextual information required to formulate an accurate design problem statement. Finally, organizational logistics dictate that systems and subsystems must be separated at established interfaces to suit the availability of resources and the appropriate domain knowledge areas of the designers involved. The collaborative team environment and the system being designed together comprise technical and social management needs. The critical item for managers to understand is the nature of the dynamics and uncertainty that are at work in the design process. They cannot be completely isolated or eliminated. The overarching management goals in the collaborative design process then become to 1) manage design iteration with the aim of producing all necessary information while mitigating "overinvestment" in volatile design areas, and 2) understand the sources of system complexity and advantageously manipulate them where possible.

\section{INFORMATION MANAGEMENT}

The design development cycle consumes project information and resources, and processes them to create the product of research and development engineering, which is all the design information necessary to realize a finished product ${ }^{7}$. Project information inputs include requirements, specifications, budget, schedule, and stakeholder influences. Examples of information outputs include design documentation, project cost information, and project status information. Additionally, material properties, stress calculations, use cases, and manufacturing cost estimates are just a few of the many types of detailed information an engineer or designer will use to formulate and refine a component design. Further time and effort will also be dedicated to producing engineering information output such as presentations for design reviews, inspection and acceptance criteria, user manuals, and assembly instructions. A vast amount of information is obviously required to formulate and deliver a complete, engineered design. It was shown in the previous section that, in the context of a system design, this quantity of information is proportional to the number of interfaces and components. In a collaborative project, barriers and impediments to effective communication can leave designers starving for relevant system information, and the inverse may lead to inundation with too numerous design objectives and constraints. This is precisely why collaborative design projects are potentially more management intensive at all levels of responsibility, versus non-collaborative projects. 
Studies suggest engineers spend somewhere between $25 \%$ and $60 \%$ of their productive time searching for and accessing the various types of information outlined above ${ }^{8,9}$; versus $10 \%$ focused on actual embodiment design details ${ }^{9}$. Conversely, nearly $90 \%$ of the situations "where the design process takes a new direction on a conceptual or embodiment design level" occur in collaborative work ${ }^{8}$. Two types of information transfer are therefore essential to satisfy interactive needs and maintain design productivity. Transactional exchanges represent a passive hand-off of information from one party or source to another, and collaborative exchanges represent bilateral participation. Detailed examples of each method as applied within the tracker upgrade project follow.

\subsection{Passive exchange: Design information transfer via PDM}

The Prime Focus Instrument Package, or PFIP, could be considered the most crucial subsystem included in the WFU by virtue of its interfaces alone. The PFIP shares its main interfaces with the tracker and it includes the Wide Field Corrector. The WFC strongback shares mechanical interfaces with the PFIP support structure, tracker hexapod, and WFC; and the WFC shares optical interfaces with the HET primary mirror and numerous scientific instruments (Figure 4). If any of these system designs differ by even the slightest amount at their interfaces, whether mechanical or optical, the HET's ability to carry out scientific research could be compromised.

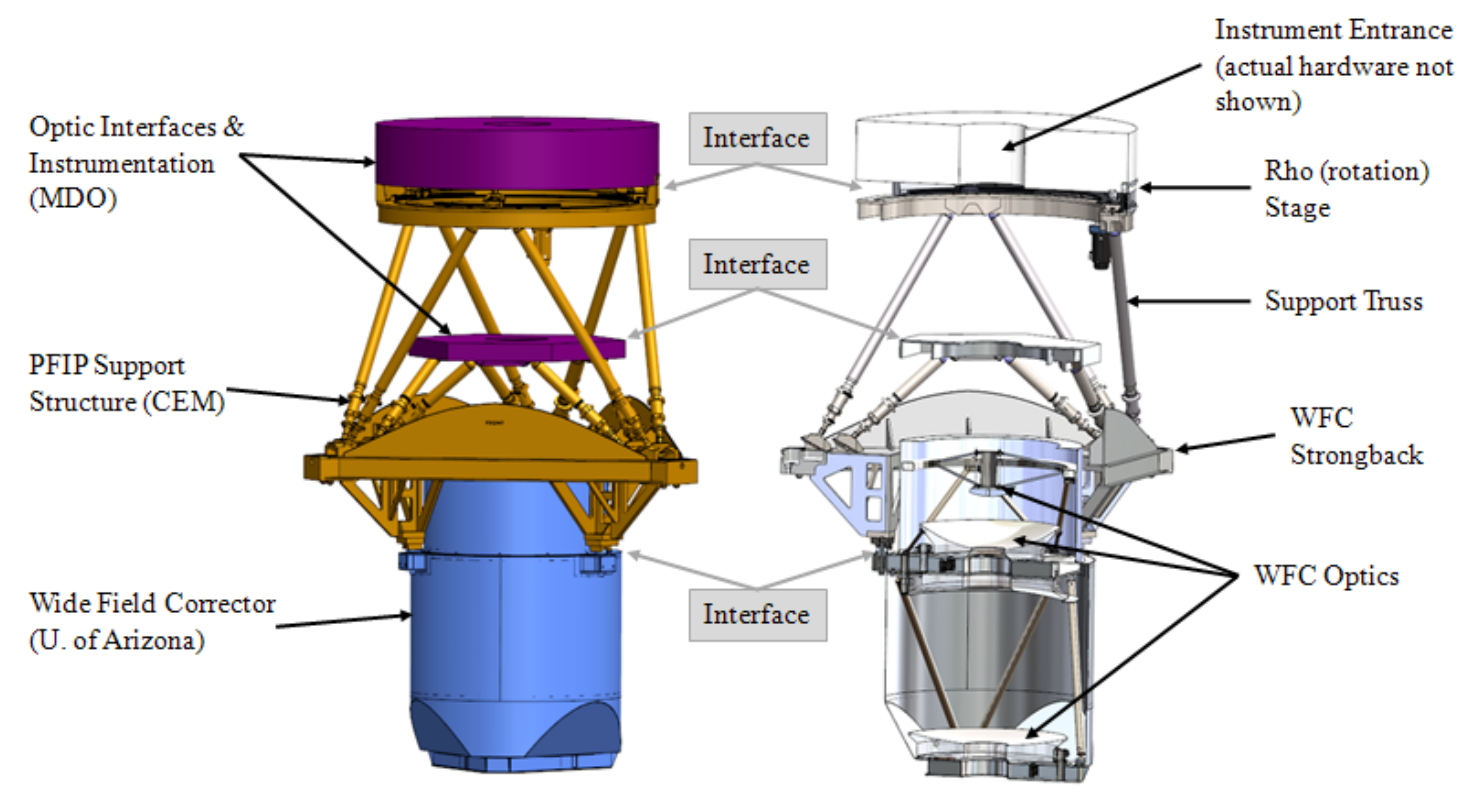

Figure 4. The Prime Focus Instrument Package (right side view is sectioned at the mid-plane) interfaces and organizational division of responsibilities (CAD content created by CEM and University of Arizona).

The interfaces that reside within the PFIP also signify some of the most critical design collaborations occurring within the project. Engineers from McDonald Observatory, the Center for Electromechanics, University of Arizona, and ADS International must all contribute the design information necessary to complete the engineering of this subsystem. Transactional and collaborative design information transfers between these parties have been critical to design progress.

Transactional information transfers are facilitated through the use of Product Data Management (PDM) software produced by the SolidWorks ${ }^{\mathrm{TM}}$ Corporation. The SolidWorks ${ }^{\mathrm{TM}}$ PDM product is integrated with the SolidWorks ${ }^{\mathrm{TM}}$ 3D Computer Aided Design (CAD) modeling environment. PDM enables networked users to upload model and design data, documentation, and virtually any format of support file e.g. presentation, spreadsheet, photos, etc. to a central fileserver referred to as the "Vault"; while also providing ownership information, user access rights, file revision control, and a number of data reporting features (see Figure 5 below). CEM has customized data collection to include supplier and cost information, delivery date, and physical inventory location as well. 


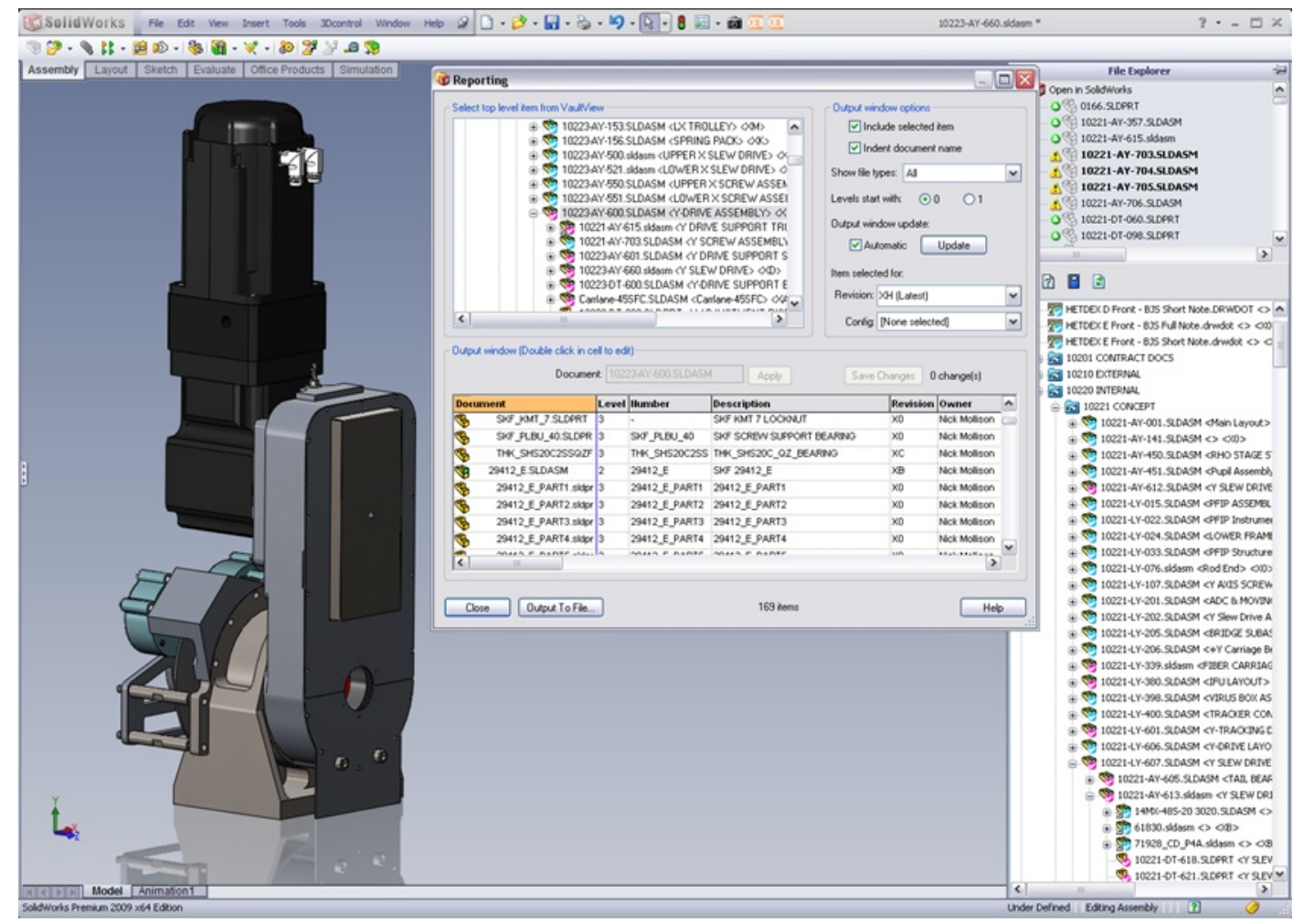

Figure 5. The SolidWorks ${ }^{\mathrm{TM}}$ PDM integrated user interface permits document reporting and tracking, and enables users to update component model information in real-time and from within the modeling environment.

The tracker design Vault is administrated by CEM. Early in the design phase, a crucial decision was made for McDonald Observatory engineers (with offices on the university main campus) working on HETDEX and WFU programs to adopt the same design software platform and share Vault access rights with CEM (located at a satellite research campus) via the University of Texas intranet. The decision to adopt a common CAD and PDM software platform has had additional benefits that extend beyond data management. Although most commercial CAD software platforms have the ability to convert model data saved in their native file format to a standardized file format (such as STEP or IGES), the accuracy of converted models is not guaranteed and the ability to exchange complete and conveniently editable models is often lost, thereby limiting their utility for collaborative work. The adoption of common design software has enabled direct engineer-to-engineer design information transfer between these two organizations. Outside organizations like the University of Arizona and ADS Intl. are indirectly linked through a slightly less efficient, but still highly effective transfer process whereby technical data packages are sent electronically to a designated point of contact within CEM, and then transferred to the Vault for use by team members. Most often this is accomplished through secure internet FTP transfer, where an FTP host address and user account is created to serve as an electronic "drop-box" for the duration of the project.

Integrating PDM with CAD has enormous benefits for parallel and collaborative design. The process of uploading CAD model data to the Vault is a many-times-daily occurrence on the tracker project. The most practical implication is that each time a design team member updates a component model in the Vault, another user working in a CAD assembly containing that component model can, in real-time, execute a "reload" command and the component will automatically update on-screen with virtually no interruption to productivity. PDM is most appropriate and most effective for this type of passive information exchange, or to transfer explicit information rather than implicit knowledge. Although notes and supporting documents can be attached to CAD information, the primary function of PDM is to enable the transfer of technically rich data in a controlled and sequential manner. It is transactional in the sense that a discrete parcel of data is packaged, transmitted, and received by one who may then process the information "as-received." Otherwise, supplemental communication such as telephone or email must be initiated in a secondary exchange. Therefore, PDM is a highly efficient tool for managing technical interchanges, but in the collaborative environment it has limitations for managing conceptually rich information and enabling participatory exchanges. 


\subsection{Participatory exchange: Failure Modes and Effects analysis}

Data from the research study introduced at the beginning of section three suggested that nearly $90 \%$ of the situations that will determine the course of a given design occur in participatory collaborations ${ }^{8}$. The next example from the tracker upgrade project recounts an event which demonstrates this conclusion. Section two of this paper made reference to the number of stakeholders and considerations that are represented in the set of tracker design objectives. One pivotal event in the tracker design process contributed more design and interface decisions in advancement toward overall design objectives than perhaps any other. This event was a Failure Modes and Effects Analysis (FMEA) conducted jointly between CEM and MDO. This face-to-face meeting commenced with both teams near-fully represented in the same conference room. The few who could not be present were able to participate via telephone and internet conferencing. The FMEA process involves agreeing upon rating scales for failure likelihood within a given time period and resulting magnitude of injury in terms of dollars, assets, or to personnel. Next, the major failure modes of each subsystem are identified, and the downstream effects of failure on the overall system are determined. In accordance with the severity of outcome and likelihood, rating scales are applied and a final risk priority rating is given to each failure mode. After the ratings have been compiled, a remediation plan for the design is constructed and individual tasks are assigned (Table 2).

Table 2. An example taken from the tracker Failure Modes and Effects Analysis exercise. Note: "RPN" refers to Risk Priority Number, which is used to prioritize risk mitigation design tasks.

\begin{tabular}{|c|c|c|c|c|c|c|c|c|c|}
\hline Item & $\begin{array}{c}\text { Potential } \\
\text { Failure Mode }\end{array}$ & $\begin{array}{l}\text { Potential } \\
\text { Effects of } \\
\text { Failure }\end{array}$ & : & $\begin{array}{c}\text { Potential } \\
\text { Cause } / \\
\text { Mechanis ms } \\
\text { of Failure }\end{array}$ & סू & $\begin{array}{l}\text { Current Design } \\
\text { Controls } \\
\text { Prevent/Detect }\end{array}$ & & RPN & $\begin{array}{c}\text { Recommended } \\
\text { Actions }\end{array}$ \\
\hline 2.1 & $\begin{array}{l}\text { Tracker bridge } \\
\text { exceeds all travel } \\
\text { limits and } \\
\text { decouples from } \\
\text { upper hexagon } \\
\text { structure. }\end{array}$ & \begin{tabular}{|l} 
Damage to \\
tracker bridge, \\
telescope \\
structure, mirrors \\
and possibly to \\
staff.
\end{tabular} & 5 & & & & & & \\
\hline 2.1 .1 & & & & $\begin{array}{l}\text { Accidental } \\
\text { power up of } \\
\text { drive motors }\end{array}$ & 3 & $\begin{array}{l}\text { - Drive motors can only be } \\
\text { powered up if both Tracker } \\
\text { Computer (TC) and } \\
\text { Telescope Control System } \\
\text { (TCS) agree (presumed) } \\
\text { - TCS detects out of } \\
\text { bounds following error and } \\
\text { commands shutdown } \\
\text { (would not apply if this is } \\
\text { not tracking event) } \\
\text { - Software limits prevent } \\
\text { tracker from exceeding } \\
\text { travel limits }\end{array}$ & 1 & 15 & $\begin{array}{l}\text { Implement torque } \\
\text { limiting device in } \\
\text { drive system. } \\
\text { TBD as to } \\
\text { whether this is } \\
\text { electrical or } \\
\text { mechanical } \\
\text { device. }\end{array}$ \\
\hline
\end{tabular}

Conducting an FMEA with the level of detail and number of parties representing the tracker design team is obviously time intensive. The full-group meeting took place over two days before splintering into assignments between pairs consisting of one CEM and one MDO engineer. As the results of the collaborative design research study would imply, the outcome of this collaboration was a number of highly important adjustments to the tracker design. Some were minor in scope, such as revisions to safety switches and control interlocks, while others redefined interfaces and added or removed whole subsystems. Another significant outcome was an overall refinement to the functional requirement definition of certain subsystems which still contained a degree of ambiguity beforehand. These types of design decision outcomes stand in contrast to those that would, or even could be enabled by passive information transfer. The heart of the FMEA was clear elicitation and explanation of design requirements, which could only take place interactively, and proportionate weighting reflects the real value of each to the various stakeholders.

\subsection{Information management summary}

One of the conclusions reached in the tracker upgrade project example is that PDM is a highly efficient tool for managing technical interchanges, but in the collaborative environment it has limitations for managing conceptually rich information and enabling interactive feedback. The implications of this last statement will become more critical to PDM- 
linked workgroups when greater degrees of system complexity are encountered. Coupling this digital sharing environment with flat organizational structures (common in many engineering and project-centric organizations) means passive information transfer will frequently occur at the lowest nodes of the network i.e. at the level of the individual responsible for a particular subsystem or component. Changes manifest in the components of the system, and each designer becomes aware of the changes as components are updated in iterative and sequential fashion. They make the newly required modifications to their component and send it to the network. If things were left this way, without supplemental communication, change propagation two or more persons removed from the change originator may be virtually invisible and therefore rarely communicated upward to the manager or to the team at-large. The tracker design team is aware of this, and conducts weekly or bi-weekly meetings where every engineer has an opportunity to announce updates and discuss work in-process.

Participatory collaboration can be time consuming and carries with it a substantial project burden when full team member presence is required. However, it is also instrumental to early collaborations that will shape the course of the design process. When such collaborative exchanges are expected to materialize ahead of time, such as design reviews and team meetings, their value should be maximized by reporting and probing architecture level design decisions to gain greater transparency into the enveloping technical and project needs, in addition to resolving details. They are also the most effective environment to initiate participatory exchanges to resolve ambiguities that may hinder design progress. Table 3 proposes a simple set of decision rules for determining an appropriate forum to address specific subject matter.

Table 3. The author's litmus test for determining medium and method of interaction based on communication needs and objectives. Reading the rows from left to right, each test is applied and the column that best describes the meeting subject matter indicates the preferred method of exchange.

\begin{tabular}{|c|c|c|c|}
\hline EXCHANGE METHOD & $\begin{array}{c}\text { PASSIVE/ } \\
\text { TRANSACTIONAL }\end{array}$ & or & $\begin{array}{l}\text { PARTICIPATORYI } \\
\text { COLLABORATIVE }\end{array}$ \\
\hline We need to exchange: & $\checkmark$ Information & & Knowledge \\
\hline That is currently: & Definite & $\checkmark$ & Uncertain \\
\hline And the content is: & $\checkmark$ Factual / Literal & & Conceptual / Abstract \\
\hline With the objective of: & Comprehension & $\checkmark$ & Agreement \\
\hline \multirow[t]{2}{*}{ And for the purpose of: } & Transfer & $\checkmark$ & Creation \\
\hline & Score $=2$ & \multicolumn{2}{|c|}{ Score $=3$} \\
\hline Exchange Method = & Passive/Transactional & \multicolumn{2}{|c|}{ Participatory/Collaborative } \\
\hline
\end{tabular}

These conclusions create the framework for the central management recommendation. Information needs and medium of communication should be considered early in the design project planning phase, and throughout the project as it evolves. It has also been shown that seeking, accessing, and processing information is a significant factor in total process time and design quality, and will therefore determine a large share of the design project's schedule and capital needs. The decision to use a common CAD and PDM platform between collaborators on the WFU program was more than a pragmatic choice. There were costs for the other party to adopt the software in the form of license fees and time required to adapt, but the costs are heavily outweighed by the rapidity and quality of design information exchange that have transpired. When uncertainty is high and/or the objective is to create information, interactive and participatory exchanges have potential to be most effective, as illustrated by the tracker FMEA example. When the inverse is true, passive and automated communication methods are suitable and likely to be most efficient.

\section{CONCLUSION}

The important conclusions contained in this paper are as follows: The selection of interfaces between components, subsystems, people, and organizations which comprise the system architecture will determine the information and communication needs of the project. Information gathering and processing is perhaps the most critical factor determining design productivity and quality. Complexity makes gathering the right information at the right time more difficult, and may obscure the impact such information will have once it is received. The challenges of managing collaborative design revolve around uncertainty and potential for hindered effort, and are compounded as the number of variable elements and stakeholders involved grow in number. Information challenges can be addressed by recognizing what needs to be 
exchanged, how the exchange should occur, and when, based on dependency relationships and whether the need is one of transfer or creation. Complexity challenges can be addressed similarly, by recognizing dependency relationships at an early stage, determining whether or not they are directional or iterative, and prioritizing to either resolve uncertainty or mitigate the strength of dependency so that designers may continue to advance their parallel development efforts.

\section{ACKNOWLEDGEMENTS}

HETDEX is led by the University of Texas at Austin McDonald Observatory and Department of Astronomy with participation from the Universitäts-Sternwarte of the Ludwig-Maximilians-Universität München, the Max-PlanckInstitut für Extraterrestriche-Physik (MPE), Astrophysikalisches Institut Potsdam (AIP), Texas A\&M University, Pennsylvania State University, and the HET consortium. In addition to Institutional support, HETDEX is funded in part by gifts from Harold C. Simmons, Robert and Annie Graham, The Cynthia and George Mitchell Foundation, Louis and Julia Beecherl, Jim and Charlotte Finley, Bill and Bettye Nowlin, Robert and Fallon Vaughn, Eric Stumberg, and many others, by AFRL under agreement number FA9451-04-2-0355, and by the Texas Norman Hackerman Advanced

Research Program under grants 003658-0005-2006 and 003658-0295-2007.

\section{REFERENCES}

[1] Savage, R. D., et al., "Current status of the Hobby-Eberly Telescope wide-field upgrade," Proc. SPIE 7733-149 (2010).

[2] Hill, G. J., Gebhardt, K., Komatsu, E., Drory, N., MacQueen, P. J., Adams, J. J., Blanc, G. A., Koehler, R., Rafal, M., Roth, M. M., Kelz, A., Gronwall, C., Ciardullo, R., Schneider, D. P., "The Hobby-Eberly Telescope Dark Energy Experiment (HETDEX): Description and Early Pilot Survey Results," ASP Conf. Series, 399, 115-118 (2008).

[3] Burge, J. H. et al, "Development of a wide-field spherical aberration corrector for the Hobby-Eberly Telescope," Proc. SPIE 7733-51 (2010).

[4] Soukup, I. M., Mollison, N. T., Mock, J. R., Beno, J. H., Hill, G. J., Good, J. M., Vattiat, B. L., Murphy, J. D., Anderson, S. C., Farenthold, E. P., Kelz, A., Roth, M. M., Bauers, S. M., "Design of the fiber optic support system and fiber bundle accelerated life test for VIRUS," Proc. SPIE, 7735-180 (2010).

[5] Heisler, J. T., Good, J. M., Savage, R. D., Vattiat, B. L, Hayes, R. J., Mollison, N. T., Soukup, I. M., "Integration of VIRUS spectrographs for the HET dark energy experiment," Proc. SPIE, 7733-153 (2010).

[6] Booth, J. A., et al., "The Wide Field Upgrade for the Hobby-Eberly Telescope," Proc. SPIE 6267, 62673W (2006).

[7] Reinertsen, D., [Managing the Design Factory], The Free Press, New York, (1997).

[8] Frankenberger, E., Badke-Schaub, P., and Birkhofer, H., Eds., [Designers: The Key to Successful Product Development], Springer, London (1998).

[9] Duffy, A., Ed., [The Design Productivity Debate], Springer, Berlin (1998).

[10] Hill, G. J., Adams, J. J., Blanc, G., Booth, J. A., Chonis, T. S., Cornell, M. E., DePoy, D. L., Drory, N., Gebhardt, K., Good, J. M., Grupp, F. U., Kelz, A., Lee, H., Marshall, J. L., MacQueen, P. J., Mollison, N. T., Murphy, J. D., Rafal, M. D., "VIRUS: a massively replicated 33k fiber integral field spectrograph for the upgraded Hobby-Eberly Telescope", Proc. SPIE 7735-21 (2010).

[11] Mollison, N. T., Mock, J. R., Soukup, I. M., Beets, T. A., Good, J. M., Beno, J. H., Kriel, H. J., Hinze, S. E., Wardell, D. R., Heisler, J. T., "Design and development of a long-travel positioning actuator and tandem constant force actuator safety system for the Hobby-Eberly Telescope wide field upgrade," Proc. SPIE, 7733-150 (2010).

[12] Worthington, M. S., Nichols, S. P., Good, J. M., Zierer, J. J., Mollison, N. T., Soukup, I. M., "Design and analysis of the Hobby-Eberly Telescope dark energy experiment (HETDEX) bridge", Proc. SPIE 7733-147 (2010).

[13] Kelz, A., Roth, M. M., Bauer, S. M., Vattiat, B., Popow, E., Padila, Y., Rambolda, W. N., Denke, M., Hayes, R. J., Hill, G. J., Good, J. M., Murphy, J. D., Savage, R. D., Soukup, I. M., Mollison, N. T., "Production and performance of replicable Integral-Field Units for VIRUS", Proc. SPIE, 7735-178 (2010).

[14] Worthington, M. S., Beets, T.A., Good, J. M., Mock, J. R., Murphy, B. T., South, B. J., "Design and development of a high precision high payload dual drive system", Proc. SPIE 7733-201 (2010). 
[15] Zierer, J. Z., Mock, J. R., Beno, J. H., Lazzarini, P. G., Fumi, P., Anaclerio, V., Good, J. M., "The development of high-precision hexapod actuators for the Hobby-Eberly Telescope Dark Energy Experiment (HETDEX)", Proc. SPIE 7733-49 (2010).

[16] Mock, J. R., Beno, J. H., Zierer, J. J., Rafferty, T. H., Cornell, M. E., “Tracker controls development and control architecture for the Hobby-Eberly Telescope dark energy experiment", Proc. SPIE 7733-152 (2010).

[17] Wedeking, G. A., Zierer, J. J., Jackson, J. R., "Kinematic optimization of upgrade to the Hobby-Eberly Telescope through novel use of commercially available three-dimensional CAD package," Proc. SPIE, 7733-148 (2010).

[18] Marshall, J. L., et al., "Production-line assembly of 150+ VIRUS spectrographs," Proc. SPIE, 7735-163 (2010).

[19] Good, J. M., Hayes, R. J., Mock, J. R., Soukup, I. M., Beno, J. H., Savage, R. D., Booth, J. A., Hill, G. J., Lee, H., Cornell, M. E., Rafal, M. D., "Design of performance verification testing for HET wide-field upgrade tracker in the laboratory," Proc. SPIE, 7739-152 (2010).

[20] Ramiller, C., Taylor, T., Rafferty, T. H., Cornell, M. E., Rafal, M. D., Savage, R. D., “A new control system hardware architecture for the Hobby-Eberly Telescope prime focus instrument package," Proc. SPIE, 7740-129 (2010).

[21] Vattiat, B. L., et al., "Mechanical design evolution of the VIRUS instrument for volume production and deployment," Proc. SPIE, 7735-264 (2010).

[22] Beno J. H., Booth, J. A., Mock, J. R., "An alternative architecture and control strategy for hexapod positioning systems to simplify structural design and improve accuracy," Proc. SPIE, 7733-97 (2010).

[23] South, B. J., Good, J. M., Booth, J. A., Worthington, M. S., Zierer, J. J., Soukup, I. M., "Wind loading analysis and strategy for deflection reduction on the HET Dark Energy Experiment upgrade,” Proc. SPIE, 7733-51 (2010).

[24] Good, J. M., Hill, G. J., Mollison, N. T., Vattiat, B. L., Murphy, J. D., Kelz, A., Roth, M. M., MacQueen, P. J., Rafal, M. D., Savage, R. D., Smith, M. P., Bayless, A. J., "Current status of the HETDEX fiber optic support system," Proc. SPIE, 7014, 7014L (2008).

[25] Savage, R. D., Booth, J. A., Gebhardt, K., Good, J. M., Hill, G. J., MacQueen, P. J., Rafal, M. D., Smith, M. P., Vattiat, B. L., "Current Status of the Hobby-Eberly Telescope Wide Field Upgrade and VIRUS," Proc. SPIE, 7012$10,(2008)$.

[26] Meiering, J. G., Buckley, D. A. H., "Southern African Large Telescope (SALT) project: progress and status after four years," Proc. SPIE, 5489, 592-602, (2004).

[27] Hill, G. J., Gebhardt, K., Komatsu, E., MacQueen, P. J., "The Hobby-Eberly Telescope Dark Energy Experiment," in The Mitchell Symposium on Observational Cosmology, AIP Conf. Proc., 743, 224, (2004).

[28] Hill, G. J., MacQueen, P. J., Palunas, P., Shetrone, M. D., Booth, J. A., "Present and Future Instrumentation for the Hobby-Eberly," Proc. SPIE, 6269, 626907 (2006).

[29] Booth, J. A., Ray, F. B., and Porter, D. S., "Development of a star tracker for the Hobby-Eberly Telescope", Proc. SPIE 3351, 298-309 (1998).

[30] Ramsey, L.W., et al., "The early performance and present status of the Hobby-Eberly Telescope," Proc. SPIE 3352, 34-42 (1998).

[31] Krabbendam, V.L., Sebring, T.A., Ray, F.B. \& Fowler, J.R., "Development and performance of Hobby-Eberly Telescope 11-m segmented mirror,” Proc. SPIE 3352, 436-445 (1998). 